\title{
FÜHRUNG
}

\section{Soziales Management}

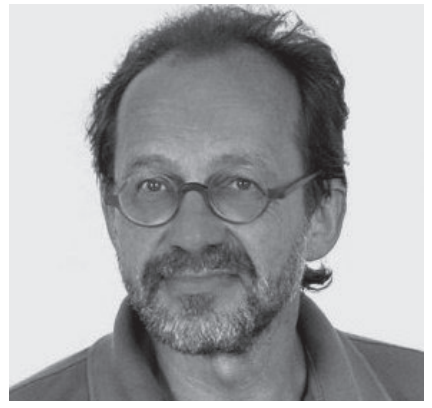

VON MICHAEL MERK

Michael Merk ist Diplom-Ingenieur und Diplom-Wirtschaftsingenieur. Nach seinem Studium war er einige Jahre als Projektmanager und Projektleiter für industrielle Großprojekte und zwanzig Jahre als Geschäftsführer von Mittelstandsund Großunternehmen tätig. www.michaelmerk.de

\author{
Durch individuelles Motivieren und soziales Management \\ können Unternehmen neue Produktivitätszuwächse \\ erreichen und Manager bessere Führungserfolge \\ erzielen. Das Ziel ist die lernende Organisation und \\ diese ist nur durch eine neue Führung zu ermöglichen.
}

Auch soziale Unternehmen können sich den veränderten Marktbedingungen nicht entziehen. Viele Manager arbeiten in einem Umfeld, das im zunehmenden Maße dadurch bestimmt ist, dass

- die für eine (Dienstleistungs-) Produktion notwendigen Input-Größen von allen Wettbewerbern zu nahezu gleichen Kosten und Qualitäten beschafft werden können

- Mitbewerber über vergleichbares Know-how verfügen

- Mitbewerber bei ähnlicher Kapitalkraft eine ähnliche Wirtschaftlichkeit der Arbeitsprozesse und damit nahezu gleiche Rentabilität erzielen

- Konkurrenten mit gleichen Produkten, denselben Zulieferern und identischen Absatzwegen agieren

- der Preis- und Konkurrenzdruck sich ständig weiter verstärkt und

- besondere Produkt- und Marktvorteile immer seltener bestehen.

- Als Folge stagnieren Umsätze und Gewinne oder sie sind gar rückläufig.

In einer solchen Situation des Wandels sind neue Führungskonzepte gesucht, die dem einzelnen Unternehmen oder dem für eine Betriebseinheit verantwortlichen Manager helfen, langfristig seine Wettbewerbsfähigkeit zu sichern und dazu beitragen, sich von anderen Marktteilnehmern abzuheben und besser als diese zu sein. Bisherige Führungskonzepte erweisen sich bei den geschilderten Marktverhältnissen immer häufiger als untauglich. Aber welche anderen Wege versprechen Erfolg, wenn weitere Entwicklungsmöglichkeiten im technisch-ökonomischen Bereich begrenzt sind?
In einer solchen Wirtschaftsphase müssen neben den eher harten ökonomischen Faktoren die "weichen « humanitätsbezogenen Sozialfaktoren eine stärkere Berücksichtigung finden. Dieser Perspektivenwechsel liegt in der ökonomischen Logik moderner Produktionsweisen sowie in der Erkenntnis, dass eine weitere Leistungssteigerung nur möglich wird, wenn der Mensch als einzigartiger Produktionsfaktor optimaler eingesetzt wird: Die im persönlichen Leistungsvermögen, in der Kreativität und in der vertrauensvollen Kooperation und Vernetzung mit anderen liegenden Ressourcen müssen ausgeschöpft werden. Der Mensch als der gestaltende Teil des Systems »Betrieb « ist allein in der Lage, die einzelnen Elemente dieses Systems zu kombinieren und ihm schöpferische Impulse zu geben.

\section{Durch Motivation erfolgreicher führen}

Dort wo Mitarbeitende aus verschiedenen Fach- und Funktionsbereichen eng zusammenarbeiten und Vorgänge und Abläufe komplexer werden, ist eine optimierte Erledigung nur durch gemeinsames Denken und Handeln, durch freiwilliges Teamwork und durch offene Kommunikation möglich. All dies gilt nicht nur für Führungskräfte, sondern gleichermaßen für Mitarbeiterinnen und Mitarbeiter sämtlicher Hierarchieebenen.

Will ein Betrieb, will ein Manager die im menschlichen Faktor schlummernden Reserven nutzen, ist es wichtig, seine Motivationen im Arbeitsleben zu kennen und hierauf einzugehen. Nur dann kann ein Management deren besondere Antriebskräfte für die betriebliche Arbeit 
wecken. Allein hierin liegt die Chance, selbst in schwierigen Zeiten erfolgreicher und besser zu werden als andere.

Es gibt eine Reihe von Führungsmitteln, die ein Unternehmen einsetzen kann, um den Leistungseinsatz seiner Mitarbeitenden zu fördern. Die Anlässe, bei denen sie eingesetzt werden können, liegen in fünf Bereichen;

- beim Verhalten der Führungskraft gegenüber seinen Mitarbeitenden

- bei der Arbeit selbst, die den Mitarbeitenden zugeordnet ist

- bei der Arbeitsplatzsituation, in der sich die Mitarbeitenden befinden

- bei der Organisation, die den Rahmen der Tätigkeit der Mitarbeitenden regelt, und

- beim Sozialklima, das in der Arbeitsumgebung herrscht.

Selbst wenn in diesen Handlungsbereichen einzelne motivationale Maßnahmen im Rahmen einer Konzeption abgestimmt und professionell umgesetzt werden, verbleibt ihnen immer der Charakter von eher sporadischen und situationsbezogenen Reaktionen.

Werden Motivationsanreize jedoch im Rahmen einer Gesamtstrategie und als Management-Konzept angewendet, entstehen Multiplikatoreffekte und Bewusstseinsveränderungen in der Belegschaft. Denn die Anreize begünstigen sich wechselseitig in ihrer Wirkung auf Motivation und Arbeitsfreude. Es kann regelrecht eine Erfolgsoffensive in Gang gebracht werden, die ansonsten nicht erreichbar wäre.

\section{Der Regelkreis des sozialen Managements}

Die langfristige Beeinflussung der persönlichen Wertvorstellungen und die Weiterentwicklung der persönlichen Reifung der Belegschaft hin zu den neuen Zielen einer leistungsförderlichen Sozialsphäre ist ohne ein Führungssystem, das von allen mitgetragen wird, und ohne die selbsterziehenden Rückkoppelungsmechanismen, die ein solches System ausmachen, nicht möglich.

Soziales Management als Führungskonzept gibt dem Unternehmen hierzu ein praktisches Regelsystem zur Hand. Führung unter dem Aspekt des sozialen Managements sucht im Kern fünf Faktoren zu verbessern:
- das häufig problembelastete Verhältnis zwischen Vorgesetzten und Mitarbeitenden

- das Verhältnis der Mitarbeitenden untereinander

- die Zufriedenheit der einzelnen Mitarbeitenden

- die mitarbeiterorientierte Denk- und Handlungsweise im Management und

- das im Betrieb vorhandene allgemeine Leistungsklima

Bei der Einführung des neuen Führungskonzepts bildet die schriftliche Formulierung von Grundsätzen und Zielen den Ausgangspunkt. Zum Einführungsbeginn müssen zunächst nur die Unternehmensphilosophie selbst und einige Anwendungsgrundsätze bestehen. Das Regelwerk wird im Verlauf der Anwendung aus sich selbst heraus wachsen - entsprechend der Wünsche der Belegschaft und gemäß der betrieblichen Erfordernisse. Diese Fortentwickelung wird zum festen Bestandteil der Führung. Im Zuge dieser Gestaltung sollten von der Betriebsleitung festgelegt werden:

- die Grundsätze einer allgemeinen Unternehmensphilosophie

- zukünftige Rollenbilder von Vorgesetzten und Mitarbeitenden sowie die hiermit verbundenen gegenseitigen Pflichten

- die Grundsätze über wesentliche betriebliche Verfahrensweisen, beispielsweise über Mitarbeitergespräche, Informationsrechte und Informationspflichten, Beurteilungen, Einführung neuer Mitarbeitender

- die Grundsätze über wesentliche organisatorische Abläufe und Regeln, beispielsweise über ein Feedbacksystem, Beurteilungswesen, Weiterbildungsmöglichkeiten, Arbeits- und Arbeitsplatzgestaltungen, Belohnungssystem, Beschwerdewesen, Vorschlagswesen

Die verschiedenen Grundsätze und Regelungen bauen aufeinander auf und stehen in einem gemeinsamen Sinnzusammenhang. Daher reicht es aus, wenn nach Formulierung der allgemeinen Unternehmensgrundsätze im weiteren sich Einzelregelungen auf das jeweilige Sachgebiet beschränken. Diese sollten nicht allzu umfangreich, dafür aber übersichtlich und leicht verständlich formuliert sein. Gegebenenfalls sollten sie durch praktische Beispiele und Erkenntnisse aus Vorkommnissen des betrieblichen Alltages erläutert und auch laufend ergänzt werden. In einem Vorwort sollten jeweils die Ziele und Vorteile der beabsichtigten Regel und der Bezug zur Unternehmensphilosophie dargestellt werden.

Jede Regel hat Zielfunktion und dient gleichermaßen als Beurteilungsmaßstab für beobachtetes Verhalten. Ihre Einhaltung muss von der Betriebsleitung kontrolliert werden und bei Nichteinhaltung muss ein Beschwerderecht gegeben sein. Bei festgestellter Missachtung müssen geeignete Interventionsmaßnahmen einsetzen. Ein solches betriebliches Normenwerk sichert, dass alle Betriebsangehörigen in Führungsfragen eine einheitliche Sprache sprechen und die persönliche Reife nach gleichen Kriterien beurteilt, entwickelt und gefördert wird. Das einmal formulierte Normenwerk muss von möglichst allen Mitarbeitenden gelebt und erlebt werden, es muss kontinuierlich überarbeitet und den betrieblichen Geschehnissen und Entwicklung angepasst werden.

Zur Optimierung der Wirkung eines sozialen Managements und zur Verbesserung der Mitarbeiterakzeptanz hat es sich bewährt, wenn die Betriebsführung sowohl bereits bei der erstmaligen Formulierung von Grundsätzen wie auch bei der weiteren Umsetzung und Fortentwicklung der Verhaltensnormen die Belegschaft einbezieht und ein beratendes Mitarbeiterteam beruft. Ein solches Präventionsgremium wirkt mit bei Aufgaben wie

- die Entwicklung von Zielen und Unternehmensgrundsätzen

- das Zusammenwirken mit der Analysestelle bei der Auswertung von Interventionen und Verbesserungsvorschlägen

- die Ableitung und Gestaltung von Grundsätzen und Richtlinien

- die Umsetzung und Kontrolle der Einhaltung

- die Konzipierung von Schulungsmaßnahmen

- die Informationsvermittelung mit Multiplikatorfunktion in der Belegschaft

Das Praktizieren eines sozialen Managements beginnt mit dem systema- 


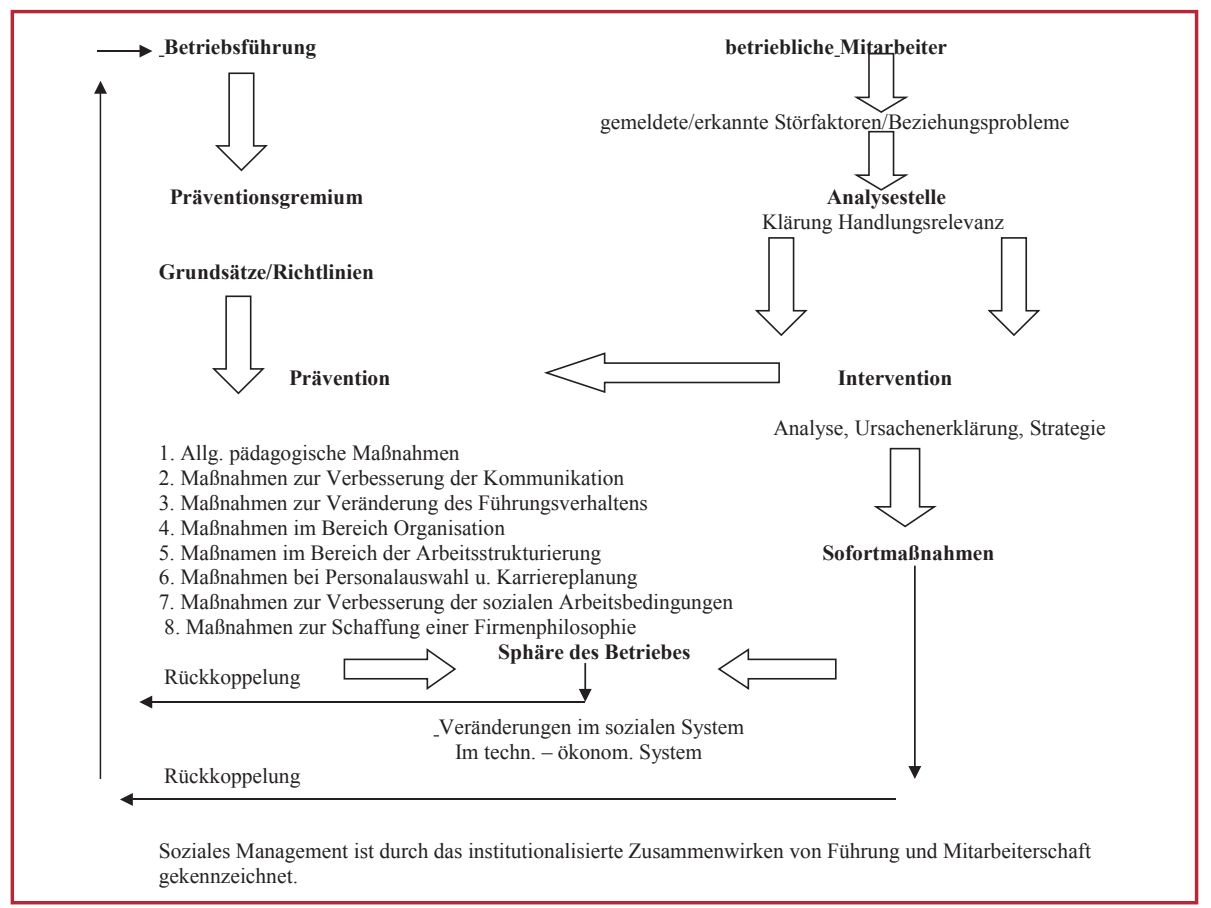

tischen Sammeln und Analysieren von leistungsbeeinflussenden Störzeichen. Eine eigene betriebliche Analysestelle ist hierfür besonders gut geeignet. Alle weitergehenden Maßnahmen dürfen nicht isoliert gesehen werden, sondern sie sind einzubetten in ein betriebliches $\mathrm{Ma}$ nagementsystem, das einerseits durch Intervention mit Sofortmaßnahmen auf konkrete Anlässe reagieren muss und das andererseits durch Prävention und erarbeitete Richtlinien in aufeinander aufbauenden Prozessen die soziale Qualität der betrieblichen Arbeitsbedingungen und Sozialbeziehungen im Gesamtbetrieb verbessert.

Dem institutionalisierten Regelprozess kommt hierbei die Aufgabe zu, Vorgesetzte wie Mitarbeitende hinsichtlich ihres Rollenverständnisses zu einem kooperativ und partnerschaftlich geprägten Verhalten hinzuentwickeln. Die Qualität dieses Regelkreises dokumentiert sich in der Zusammenarbeit der beteiligten Institutionen und in der gemeinsamen Gestaltung des betrieblichen Regelwerkes (vgl. Abbildung).

Erfolgreiche Leistungsmotivierung ist als fortwährender Managementprozess zu verstehen. Wird in einem Unternehmen ein Regelkreis entsprechend der hier geschilderten Führungsprinzipien eingeführt und gelebt, setzt bei den Menschen selbst wie auch bei den betrieblichen Arbeitsbedingungen ein Veränderungsprozess ein, an dessen Ende leistungshemmende Störfaktoren im Rahmen des wirtschaftlich Möglichen abgebaut sind. Es werden Strukturen geschaffen, die den gemeinsamen Bedürfnissen entsprechen und die als Maßstäbe für Verhaltensweisen anerkannt werden. Es entsteht eine lebende Organisation, in der zielgerichtete Veränderungsprozesse zur üblichen Praxis werden. Die Rückkoppelungsmechanismen in dem beschriebenen Regelkreis bewirken ein lernfähiges System, das sich fortwährend weiterentwickelt.

\section{Die Zunahme der sozialen Reife}

- bewirkt verbessertes Sozialverhalten: Kommunikative und partnerschaftliche Verhaltensweisen werden allmählich zur Selbstverständlichkeit.

- hat Einfluss auf das aufgabenbezogene Verhalten: Mit zunehmender persönlicher Reifung steigt der Wille, die Befähigung und das Selbstvertrauen der Mitarbeitenden spezifische Funktionen und Aufgaben selbstständig zu übernehmen. Bei bedürfnisgerechter Ausgestaltung der Arbeit steigt ihr Eigeninteresse hieran, denn ihr Wunsch nach Erfüllung von selbstbezogenen Bedürfnissen wächst.

- bewirken bei den Vorgesetzten eine Veränderung ihrer Wertvorstellungen und ein verändertes Führungsverhalten: Der Führungsstil wandelt sich von einem stark aufgabenbezogenen und vielleicht autoritär ausgerichteten Führungsverhalten zu einem Organisationsmanagement, das die persönlichen Belange der Mitarbeitenden stärker berücksichtigt und diese angemessen in die Entscheidungsprozesse einbezieht.

- übt einen Veränderungsdruck aus auf die Gestaltung und Organisation der Arbeit: In dem Maße wie aufgabenbezogenes Denken und Verhalten sich verändern, nehmen auch die Möglichkeiten, die Bereitschaft und das Verlangen $\mathrm{zu}$, betriebliche Situationen entsprechend anzupassen.

\section{Was wirklich erreichbar ist}

Durch das vorgestellte Führungsmodell können Motivationskräfte gesteigert und die Leistungen von Mitarbeitenden erheblich verbessert werden. Im Zeitalter der Arbeitsteilung ergeben sich Erfolge aber nicht allein aus solchen Leistungssteigerungen beim einzelnen Mitarbeitenden. Immer öfter sind sie ein Ergebnis guter Zusammenarbeit mehrerer und zumeist das Resultat einer gemeinschaftlichen Kooperation verschiedener organisatorischer Betriebseinheiten. So können durch das Führungsmodell nutzbare Multiplikatoreffekte für die betriebliche Produktivität entstehen und es entwickelt sich eine positive betriebliche Dynamik. Wird der skizzierte Regelkreis im Bereich der Analysestelle um einen technischen Fachbereich ergänzt, kann gezielt die Belegschaft auch in die technische Optimierung von Arbeitsabläufen einbezogen werden.

Das insgesamt durch das beschriebene Führungsmodell sich neu erschließende Erfolgspotenzial ist von Unternehmen zu Unternehmen unterschiedlich. In jedem Falle ist es gravierend und in der augenblicklichen Situation des Umbruchs wird es vielfach zukunftsentscheidend sein.

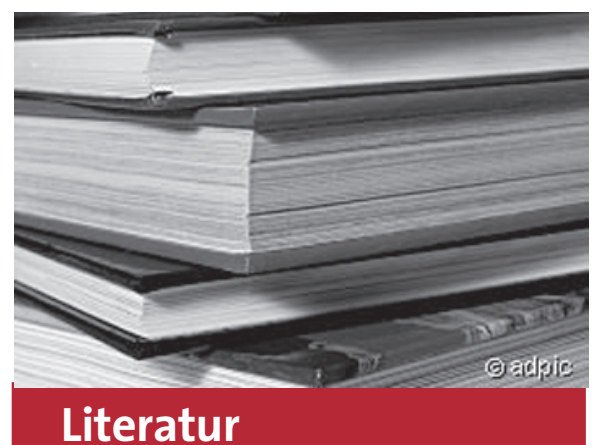

Michael Merk: Warum manche Manager Erfolg haben und andere immer erfolglos bleiben. Books on Demand GmbH, Norderstedt 2008. 292 Seiten. 20,80 Euro. ISBN 978-3-8334-8474-2. 